\title{
How to Improve the Efficiency of Hospital Administration under the new Situation of Medical Reform \\ Ning-Can LONG
}

The First Affiliated Hospotal, Jinan University, Guangzhou 1413615985@qq.com

Key words: Medical reform, Hospital administration, Efficiency.

\begin{abstract}
At present, the comprehensive reform of public hospitals in the province has been completely pushed aside, and the large-scale reform has put forward higher requirements for the administration of the current hospitals. Through the analysis of the current situation and existing problems of administrative management, the methods to improve the efficiency of hospital administration under the new medical reform situation are explored.
\end{abstract}

\section{Introduction}

In July 15, 2017, the comprehensive reform of public hospitals in Guangdong province has been fully pushed open. With the deepening of the health system, the medical institutions among peers facing more intense competition, therefore, reasonable construction management personnel, administrative personnel play a role, improve the management of the comprehensive quality and innovation ability, is the key to enhance the efficiency of hospital management.

\section{Problems in the Management Personnel of Hospital}

Hospital administrators are managers of all departments. Their own ability and level determine not only the performance of the leading department, but also the continuous, stable and healthy development of the hospital [1]. At present, the main source of hospital administrative staff of medical personnel in "business transformation" from the physicians, nurses, medical departments transferred to administrative positions; although some is also in charge of the work unit, has abundant clinical experience, but they have no formal public hospital management training, lack of administrative management level of hospital scientific management, therefore the more traditional mode and limitations. In addition, some comrades who just graduated from colleges and universities and go directly to administrative management positions, though highly educated, have strong language skills, accept new things, new knowledge and new ideas, but lack social experience and practical experience. Most of the senior managers in hospitals are at the same time as medical technology backbone or academic leaders in some disciplines. They have high popularity and authority in a medical field, which is conducive to the scientific construction and international exchange of hospitals. However, because of their high level management, because of various affairs, they lack the time to communicate with their subordinates and the macro thinking of scientific management. Therefore, there is a universal existence of a single knowledge structure and the experience based management, which restricts the improvement of the level of hospital management.

\section{The Lack of a Scientific and Reasonable Regulatory System}

The administrative work of the hospital has the characteristics of large amount of business, strong ability to strain, complicated work and strong policy. No hospital administrative staff will be the work performance, work quality, work efficiency and bonuses; annual assessment appraised also can not play a fundamental role in motivation; common mess phenomenon, most of that hospital management work belongs to the daily routine work, able to cope with it, ganhaoganhuai a kind, no high demands on their own. 
At present, it is particularly urgent to strengthen the construction of hospital administrative staff, improve the comprehensive quality of hospital administrators, and improve the efficiency of hospital administration [2].

\section{Ways to Improve the Efficiency of Administrative Work}

1) The hospital administration should follow the principle of basic concept of human relations, human nature, humanity, human rights, scientific and reasonable number of posts, assign tasks according to the work characteristic of each department is reasonable, pay attention to clear responsibilities, improve the responsibility to implement the organizational leadership mechanism, and make sure the next one tube, everything was caught, each piece is implemented the work pattern, carefully organize the administrative staff of positive action, threw himself into the work, to ensure that each work carried out, together to the hospital to become bigger and stronger. In a word, must pay attention to the selection, education and innovation mechanism of the establishment of personnel can be hired, promoted or demoted, post treatment can lower energy, reasonable personnel structure, full of vigor and vitality of the operating mechanism, so as to establish a high quality business good occupation management team[3].

2) Strengthening the training of professional knowledge of management is the only way to improve the quality of personnel. Hospitals should provide opportunities and conditions as far as possible, so that administrators can use "spare time" to "charge" regularly, and organize administrative staff to participate in business training and related practice assessment regularly. Only by constantly enhancing learning can we make administrators accumulate a wide range of knowledge of hospital management, so that we can have pioneering spirit, excellent management and organizational coordination.

3) It is helpful to stimulate and arouse the enthusiasm of managers to improve the incentive mechanism and enhance the effective incentive mechanism. Hospital leaders should link work performance, work quality and work efficiency with administrative staff's bonus and Professional Title Review, encourage and guide managers to take the initiative, keep forging ahead and keep pace with the times, so as to improve work efficiency. At the same time, the administrative process should be infiltrated into the clinical department's year-end summary, subject assessment, professional promotion, tenure evaluation, and so on, so as to avoid the distraction of clinical departments for these non clinical work[4].

4)Administrative staff should adhere to the principle of "hospital is my home, innovation by everyone", let "mobilize me to do" become "I do". The work should pay attention to "strict" and "hard", "true", strengthen the responsibility to implement measures to ensure effectiveness; in doing fine work on, and make the effort in the United States; learn good practices and experience, to further focus the work, to increase efforts to promote and improve the work standard. To promote the development of hospital.

\section{Conclusion}

In short, the construction of new era hospital is inseparable from efficient administrative management, constantly improving the administrative efficiency of hospitals, and will play an important role in promoting and promoting the development of hospitals[4]. Strengthening the democratic management level, promoting the healthy development of the hospital, enhancing the sense of responsibility of the medical staff, mobilizing the enthusiasm of the staff and promoting the reform and development of the hospital are the key to improve the administration of the hospital. Administrative staff is the executor of policy decision in hospitals. The quality of their work directly affects the image, social benefits, economic benefits and internal safety of hospitals, which can play a decisive role in the development of hospitals. The urgent task is to strengthen the construction of administrative staff, improve the comprehensive quality, update the management concept, emphasize the management methods, improve the efficiency of hospital management, 
enhance the brand image of the hospital, promote the steady improvement of medical quality, and maintain the healthy and rapid development of the hospital.

\section{References}

[1] Xiao Xiantao. Practice of hospital management staff assessment J. Chinese hospital management, 2000, 20 (6): 34-35.

[2] Zhou Xiaoling, Li Weiming. The reform and effectiveness of the system of selection and appointment for middle level cadres in our hospital [J]. Chinese hospitals, 2005, 9 (5): 43-44.

[3] Wang Lin. A brief discussion on the construction of the administrative personnel team of hospital administration [J]. cooperation economy and science and technology, nineteenth phase of November 2010.

[4] Zheng Yansong, Ouyang Yadi, Chen Zhilai, Wuqiang, Fan Li. Talk about the optimization of hospital administrative processes [J]. Journal of Chinese people's Liberation Army hospital management, October 2009 sixteenth. 